MATHEMATICS OF COMPUTATION

Volume 71, Number 240, Pages 1545-1567

S 0025-5718(01)01389-8

Article electronically published on August 3, 2001

\title{
LONG-TERM STABILITY \\ OF VARIABLE STEPSIZE APPROXIMATIONS OF SEMIGROUPS
}

\author{
NIKOLAI BAKAEV AND ALEXANDER OSTERMANN
}

\begin{abstract}
This paper is concerned with the stability of rational one-step approximations of $C_{0}$ semigroups. Particular emphasis is laid on long-term stability bounds. The analysis is based on a general Banach space framework and allows variable stepsize sequences. Under reasonable assumptions on the stepsize sequence, asymptotic stability bounds for general $C_{0}$ semigroups are derived. The bounds are typical in the sense that they contain, in general, a factor that grows with the number of steps. Under additional hypotheses on the approximation, more favorable stability bounds are obtained for the subclass of holomorphic semigroups.
\end{abstract}

\section{Introduction}

Stability bounds for approximations of linear semigroups play, without doubt, a key role in the analysis of discretizations of evolution equations. Consequently, such bounds have gained a lot of interest in literature (see, e.g., Bakaev [1], Brenner and Thomée [4, LeRoux [9], Lubich and Nevanlinna [10], and Palencia [13]). Whereas these stability bounds apply directly in the analysis of linear equations, their importance for nonlinear problems relies on the use of perturbation techniques and the variation-of-constants formula (cf., e.g., Lubich and Ostermann [11]).

In the present paper, we are concerned with the long-term behavior of rational approximations to $C_{0}$ and in particular holomorphic semigroups in the case of nonuniform time grids. It is well known that the use of nonconstant stepsize sequences in the numerical treatment of differential equations may lead to an asymptotic behavior of the approximate solution which essentially differs from that of the exact solution. For systems of ordinary differential equations (linear and nonlinear) the analysis of asymptotic stability of approximate solutions on nonuniform grids is carried out in a paper by Hairer and Zennaro [6]. The results of [6] can be applied to the case of linear differential equations in Hilbert spaces with normal operators. However, in Banach spaces, the situation is much more complicated and a more careful analysis is required.

It was shown in Bakaev [2] and later in Palencia [13] that, under some reasonable restrictions, rational approximations of a bounded holomorphic semigroup are uniformly bounded for arbitrary stepsize sequences. For holomorphic semigroups

Received by the editor July 10, 2000 and, in revised form, December 26, 2000.

2000 Mathematics Subject Classification. Primary 65M12, 65L20.

The work of the first author was supported by the Swiss National Science Foundation under Grant 20-56577.99.

The second author was on leave from Universität Innsbruck, Institut für Technische Mathematik, Geometrie und Bauinformatik, Technikerstraße 13, A-6020 Innsbruck, Austria. 
with exponential decay, however, the asymptotic behavior of corresponding approximations does not seem to have been studied earlier.

As concerns general $C_{0}$ semigroups, we are also not aware of any work devoted to the analysis of approximations on nonuniform grids, particularly not for semigroups with exponential decay. However, we have to mention the seminal paper by Brenner and Thomée 4, where approximations of $C_{0}$ semigroups with constant time steps are examined, and on which our analysis will be based.

Let $E$ be a Banach space with norm $\|\cdot\|$. The symbol $\|\cdot\|$ also denotes the operator norm in $E$. Consider in $E$ the following Cauchy problem

$$
u_{t}=A u, \quad 0<t<\infty ; \quad u(0)=u_{0},
$$

with some linear unbounded operator $A$ acting on $E$ and some initial value $u_{0} \in E$.

Our main hypothesis with respect to the operator $A$ will be as follows:

HA1: The operator A generates a $C_{0}$ semigroup $e^{t A}$, and there are constants $L>0$ and $\nu \geq 0$ such that

$$
\left\|e^{t A}\right\| \leq L e^{-\nu t} \quad \text { for all } t \geq 0 .
$$

Hypothesis HA1 allows one to define the mild solution of problem (1) by

$$
u(t)=e^{t A} u_{0}, \quad t \geq 0 .
$$

It is well known that HA1 can be replaced by an equivalent condition in terms of the resolvent of the operator $A$ (see, e.g., Hille and Phillips [8]) but this is not essential for our further needs. Nevertheless, in the last section, we shall encounter the following sectorial type restriction on $A$ :

HA2: The operator $A$ is closed, with dense domain and its resolvent satisfies

$$
\left\|(z I-A)^{-1}\right\| \leq L|z+\nu|^{-1} \quad \text { whenever } z \in \Sigma_{\chi \nu},
$$

with some $L>0, \chi \in(0, \pi / 2)$, and $\nu \geq 0$, where

$$
\Sigma_{\chi \nu}=\{z \in \mathbb{C}: z \neq-\nu,|\arg (z+\nu)| \leq \pi-\chi\} \cup\{-\nu\} .
$$

We note that hypothesis HA2 is more restrictive than HA1 because, if it is fulfilled, $A$ generates a holomorphic $C_{0} \operatorname{semigroup~} e^{t A}$, with

$$
\left\|e^{t A}\right\| \leq C L e^{-\nu t} \quad \text { for all } t \geq 0
$$

where the constant $C$ depends on $\chi$ (see, e.g., Lunardi [12, Chapter 2]).

We next introduce our hypotheses on the rational approximations. Let $R(z)$ denote a fixed rational function. We require $R(z)$ to be $A(\varphi)$-stable, i.e., HR1: $|R(z)| \leq 1$ for all $z \in \mathbb{C} \backslash \Sigma_{\varphi 0}$, with some $\varphi \in(0, \pi / 2]$.

With the function $R(z)$ we associate the following function

$$
r(\xi, \eta)=R(\eta+i \xi), \quad \xi, \eta \in \mathbb{R},
$$

and we shall write for brevity,

$$
r(\xi)=R(i \xi) .
$$

Sometimes we shall use the following additional restrictions on $R(z)$ :

HR2: The number $R_{\infty}=R(\infty)$ satisfies

$$
\left|R_{\infty}\right|<1
$$

HR3: The function $r(\xi)$ fulfils

$$
|r(\xi)|<1 \quad \text { for all } \xi \in \mathbb{R} \backslash\{0\} ;
$$


and

HR4: $R(z)$ approximates $e^{z}$ at $z=0$ with order $p$ for some $p \geq 1$, that is

$$
R(z)=e^{z}+\mathcal{O}\left(|z|^{p+1}\right) \text { as } z \rightarrow 0 .
$$

It follows from the hypotheses HR2, HR3, and HR4 that

HR5: The function $a(\xi)=e^{-i \xi} r(\xi)$ satisfies for any fixed $d>0$

$$
|a(\xi)| \leq e^{-c \xi^{s}} \quad \text { whenever }|\xi| \leq d
$$

with some even $s \geq p+1$ and some $c>0$ (depending on $d$ ).

However, the size of $s$ is not specified by HR2 -4 and we prefer to introduce HR5 as a separate assumption.

Throughout this paper, we deal with sequences of positive numbers. For brevity, we call each such sequence a stepsize sequence. For any stepsize sequence $K=$ $\left(k_{j}\right)_{j=1}^{\infty}$, we define the finite stepsize sequence $K_{n}=\left(k_{j}\right)_{j=1}^{n}$, whose members are just the $n$ first members of $K$. In this case, we write $K_{n} \subset K$.

For any finite stepsize sequence $K_{n}=\left(k_{j}\right)_{j=1}^{n}$, we introduce the operator

$$
R\left(K_{n} ; A\right)=\prod_{j=1}^{n} R\left(k_{j} A\right)
$$

which may be thought of as a rational variable stepsize approximation to the semigroup $e^{t_{n} A}$ with $t_{n}=\sum_{j=1}^{n} k_{j}$. The main aim of the present paper is thus to obtain bounds for the quantity $\left\|R\left(K_{n} ; A\right)\right\|$, under some reasonable restrictions on $A$ and $R(z)$ from among those listed above. In particular, we examine the long-term behavior of rational methods of the form (3).

The paper is organized as follows. In Section 2, we consider the case when the operator $A$ fulfils HA1 with $\nu=0$ and, therefore, generalize results of Brenner and Thomée [4] on stability to the case of variable time steps. Section 3 is devoted to the case $\nu>0$ and contains results which allow one to compare the asymptotic behavior of the semigroup $e^{t A}$ to that of the rational method (3). Our analysis in these two sections is based on the Hille-Phillips operator calculus. Finally, in Section 4, we examine asymptotic stability in the case of holomorphic semigroups, that is when the operator $A$ fulfils HA2 with $\nu>0$. We obtain in this case sharper estimates of asymptotic stability. The techniques for showing this are based on the Dunford operator calculus in terms of resolvents.

For our further needs, it is convenient to introduce some additional notation. For any given finite stepsize sequence $K_{n}=\left(k_{j}\right)_{j=1}^{n}, n \geq 1$, we denote for $\beta \neq 0$,

$$
S_{\beta}\left(K_{n}\right)=\left(\sum_{l=1}^{n} k_{l}^{\beta}\right)^{1 / \beta} \text { and } M_{\beta}\left(K_{n}\right)=n^{-1 / \beta} S_{\beta}\left(K_{n}\right)
$$

As above, instead of $S_{1}\left(K_{n}\right)$, we simply write $t_{n}$.

With any finite stepsize sequence $K_{n}=\left(k_{j}\right)_{j=1}^{n}$, we associate the reordered stepsize sequence $H_{n}=\left(h_{j}\right)_{j=1}^{n}$, given by $h_{j}=k_{\sigma(j)}$, where the bijection $\sigma$ is chosen in a way such that

$$
h_{1} \leq \ldots \leq h_{n}
$$

The finite sequence $H_{n}$ is called the ordered sequence for $K_{n}$. 
Next let $K_{n}=\left(k_{j}\right)_{j=1}^{n}$ be an arbitrary finite stepsize sequence and let $H_{n}=$ $\left(h_{j}\right)_{j=1}^{n}$ be the corresponding ordered stepsize sequence. Let the indices

$$
1 \leq l_{1} \leq \ldots \leq l_{\mu}=n
$$

be chosen such that $l_{j}, 1 \leq j \leq \mu-1$ is the largest index for which $2 h_{l_{j}}<h_{l_{j+1}}$, and $2 h_{1} \geq h_{l_{1}}$. The finite sequence $\left(l_{j}\right)_{j=1}^{\mu}$ is called the associated sequence of indices for $K_{n}$.

For any finite stepsize sequence $K_{n}=\left(k_{j}\right)_{j=1}^{n}$, we denote

$$
S_{j, \beta}\left(K_{n}\right)=\left(\sum_{l=1}^{l_{j}} h_{l}^{\beta}\right)^{1 / \beta}, j=1, \ldots, \mu,
$$

and

$$
M_{j, \beta}\left(K_{n}\right)=l_{j}^{-1 / \beta} S_{j, \beta}\left(K_{n}\right), \quad j=1, \ldots, \mu,
$$

where $\left(h_{j}\right)_{j=1}^{n}$ is the ordered finite sequence for $K_{n}$ and $\left(l_{j}\right)_{j=1}^{\mu}$ the associated sequence of indices for $K_{n}$.

In the case when $K_{n}$ is specified in the context, we shall often omit the dependence of $S_{\beta}, M_{\beta}, S_{j, \beta}$, and $M_{j, \beta}$ on $K_{n}$.

For future reference, we recall the following well-known inequalities (for their proofs, see, e.g., Hardy, Littlewood, and Pólya [7]) :

$$
\begin{array}{cl}
S_{\beta_{2}}\left(K_{n}\right) \leq S_{\beta_{1}}\left(K_{n}\right) & \text { for } 0<\beta_{1} \leq \beta_{2}, \\
M_{\beta_{1}}\left(K_{n}\right) \leq M_{\beta_{2}}\left(K_{n}\right) & \text { for } \beta_{1} \leq \beta_{2} .
\end{array}
$$

Obviously, the inequalities (4) and (5) are valid with $S_{j, \beta}\left(K_{n}\right)$ and $M_{j, \beta}\left(K_{n}\right)$ substituted for $S_{\beta}\left(K_{n}\right)$ and $M_{\beta}\left(K_{n}\right)$, respectively.

Henceforth, we denote by $C$ and $c$ generic constants, subject to $C \geq 0$ and $c>0$, whose sizes are not essential for our analysis. We emphasize that these constants depend only on the constants appearing in our hypotheses, except on $L$. The dependence on $L$ will always be given explicitly.

\section{Bounded SEMigroups}

In this section, we show how the fundamental results of Brenner and Thomée 4 on the stability of rational approximations of semigroups can be extended to the case of nonconstant stepsize sequences. Note that for constant stepsizes, the estimates (28) and (29) below were obtained already in [4].

We begin with some preliminaries. Let $\Lambda$ be the set of bounded measures $\lambda(t)$ on the real axis $\mathbb{R}$ and let $\widehat{\Lambda}$ be the set of corresponding Fourier transforms

$$
\widehat{\lambda}(\xi)=\int_{-\infty}^{\infty} e^{i t \xi} d \lambda(t), \quad \lambda \in \Lambda .
$$

Endowed with the norm

$$
\llbracket \hat{\lambda} \rrbracket=\int_{-\infty}^{\infty} d|\lambda|(t)
$$

where $\hat{\lambda}$ and $\lambda$ are connected via (6) , the set $\widehat{\Lambda}$ becomes a Banach algebra, so that

$$
\llbracket \hat{\lambda}_{1} \hat{\lambda}_{2} \rrbracket \leq \llbracket \hat{\lambda}_{1} \rrbracket \llbracket \hat{\lambda}_{2} \rrbracket \text { for all } \hat{\lambda}_{1}, \hat{\lambda}_{2} \in \widehat{\Lambda} \text {. }
$$


As a generalization of formula (6) to the operator case, Hille and Phillips [8, Chapter XV] have constructed a calculus for a certain class of generators of semigroups. Within this framework, they have derived as well an estimate for the norm of a function of such an operator (see [8, Theorem 15.2.1]). For further needs, we use the following straightforward consequence of this estimate: if a function $F(z)$ is holomorphic and bounded in the half-plane $\operatorname{Re} z \leq 0$ with $f(\xi)=F(i \xi) \in \widehat{\Lambda}$, and if the operator $A$ fulfils hypothesis HA1 with $\nu=0$ and some $L>0$, then

$$
\|F(A)\| \leq L \llbracket f \rrbracket .
$$

On the other hand, the quantity $\llbracket f \rrbracket$ can be bounded by Carlson's inequality (cf. [4, Lemma 2]):

$$
\llbracket f \rrbracket \leq \sqrt{2}\|f\|_{L_{2}}^{1 / 2}\left\|f^{\prime}\right\|_{L_{2}}^{1 / 2} \quad \text { whenever } f, f^{\prime} \in L_{2} .
$$

Our analysis below is essentially based on the use of (8) and (9). Let $K_{n}=$ $\left(k_{j}\right)_{j=1}^{n}$ be an arbitrary finite stepsize sequence. Here and elsewhere in this section, given $K_{n}$, we denote

$$
P(\xi)=\prod_{l=1}^{n} r\left(k_{l} \xi\right) .
$$

If $R(z)$ fulfils hypothesis $\mathbf{H R} 1$ with $\varphi=\pi / 2$, the function $\prod_{l=1}^{n} R\left(k_{l} z\right)$ is holomorphic and bounded in the half-plane $\operatorname{Re} z \leq 0$. Further, in this case, as shown in [4], $r(\xi) \in \widehat{\Lambda}$, and hence by Lemma 6 in [4], $r(k \xi) \in \widehat{\Lambda}$ for any $k>0$. Since $\widehat{\Lambda}$ is a Banach algebra, we conclude that $P(\xi) \in \widehat{\Lambda}$. Therefore, as a consequence of (프), we obtain

$$
\left\|R\left(K_{n} ; A\right)\right\| \leq L \llbracket P(\xi) \rrbracket .
$$

Together with formula (99), this yields the basis for our subsequent analysis.

Our first result on stability is as follows:

Theorem 2.1. Let the operator $A$ and the function $R(z)$ satisfy HA1 with $\nu=0$, $L>0$ and $\mathbf{H R} 1$ with $\varphi=\pi / 2$. Then, for any finite stepsize sequence $K_{n}=\left(k_{j}\right)_{j=1}^{n}$, we have

$$
\left\|R\left(K_{n} ; A\right)\right\| \leq C L \varepsilon^{-1} n^{1 / 2}\left(\frac{M_{1 / 2}\left(K_{n}\right)}{M_{-\varepsilon}\left(K_{n}\right)}\right)^{\varepsilon /(4 \varepsilon+2)} \quad \text { for all } \varepsilon \in(0,1] .
$$

Proof. We shall bound the right-hand side of (10). Let $k$ be an arbitrary positive number. For further needs, it is convenient to denote $\kappa_{l}=k_{l} / k$ and

$$
P(k ; \xi)=\prod_{l=1}^{n} r\left(\kappa_{l} \xi\right) .
$$

It follows from the above remarks that $P(\xi) \in \widehat{\Lambda}$ and hence, by Lemma 6 in [4],

$$
\llbracket P(\xi) \rrbracket=\llbracket P(k ; \xi) \rrbracket .
$$

As will be seen below, a suitable bound for the right-hand side of (12) and the optimal choice of the parameter $k>0$ will lead to the desired estimate (11).

Let $\psi(\xi)$ be a smooth cut-off function satisfying the conditions

$$
\text { supp } \psi \subset\left(-2,-\frac{1}{2}\right) \cup\left(\frac{1}{2}, 2\right) \quad \text { and } \quad \sum_{j=1}^{\infty} \psi\left(2^{-j} \xi\right)=1 \quad \text { if }|\xi|>2 \text {. }
$$


The existence of such a function is shown in Brenner, Thomée, and Wahlbin [5. Lemma 2.1.1]). Denote

$$
\psi_{j}(\xi)=\psi\left(2^{-j} \xi\right), \quad j=1,2, \ldots, \quad \text { and } \quad \psi_{0}(\xi)=1-\sum_{j=1}^{\infty} \psi_{j}(\xi) .
$$

Clearly, the family $\left(\psi_{j}\right)_{j=0}^{\infty}$ is a partition of unity. By the triangle inequality, we can write

$$
\llbracket P(k ; \xi) \rrbracket \leq \llbracket R_{\infty}^{n} \rrbracket+\sum_{j=0}^{\infty} \llbracket \psi_{j}(\xi)\left(P(k ; \xi)-R_{\infty}^{n}\right) \rrbracket .
$$

For further success, we need the following simple inequalities which are valid for all $\xi \in \mathbb{R}$,

$$
\begin{gathered}
|r(\xi)| \leq 1 \\
\left|r(\xi)-R_{\infty}\right| \leq C(1+|\xi|)^{-1},
\end{gathered}
$$

and

$$
\left|\frac{d}{d \xi} r(\xi)\right| \leq C(1+|\xi|)^{-2} .
$$

By applying (14) and (15), we find

$$
\begin{array}{r}
\left|P(k ; \xi)-R_{\infty}^{n}\right| \leq \sum_{l=1}^{n}\left|R_{\infty}\right|^{l-1}\left|r\left(\kappa_{l} \xi\right)-R_{\infty}\right| \prod_{j=l+1}^{n}\left|r\left(\kappa_{j} \xi\right)\right| \\
\leq C \sum_{l=1}^{n}\left(1+\kappa_{l}|\xi|\right)^{-1} \quad \text { for all } \xi \in \mathbb{R} .
\end{array}
$$

The last inequality can be sharpened when taking into account that by (14),

$$
\left|P(k ; \xi)-R_{\infty}^{n}\right| \leq 2 \text { for } \xi \in \mathbb{R},
$$

so that we get instead of (17),

$$
\left|P(k ; \xi)-R_{\infty}^{n}\right| \leq C \min \left(1, \sum_{l=1}^{n}\left(1+\kappa_{l}|\xi|\right)^{-1}\right) \quad \text { for all } \xi \in \mathbb{R} .
$$

One can also obtain on the basis of (14) and (16) for all $\xi \in \mathbb{R}$,

$$
\begin{array}{r}
\left|\frac{d}{d \xi}\left(P(k ; \xi)-R_{\infty}^{n}\right)\right| \leq \sum_{l=1}^{n} \kappa_{l}\left|\frac{\partial}{\partial \xi} r\left(\kappa_{l} \xi\right)\right|\left|\prod_{j \neq l} r\left(\kappa_{j} \xi\right)\right| \\
\leq C \sum_{l=1}^{n} \kappa_{l}\left(1+\kappa_{l}|\xi|\right)^{-2} .
\end{array}
$$

Now, by using (18) and (19) and taking into account how the support of each $\psi_{j}$ is localized, we get for $j \geq 1$,

$$
\left\|\psi_{j}(\xi)\left(P(k ; \xi)-R_{\infty}^{n}\right)\right\|_{L_{2}} \leq C 2^{j / 2} \min \left(1, \sum_{l=1}^{n}\left(1+\kappa_{l} 2^{j}\right)^{-1}\right),
$$


and

$$
\left\|\frac{d}{d \xi}\left(\psi_{j}(\xi)\left(P(k ; \xi)-R_{\infty}^{n}\right)\right)\right\|_{L_{2}} \leq C\left(2^{-j / 2}+2^{j / 2} \sum_{l=1}^{n} \kappa_{l}\left(1+\kappa_{l} 2^{j}\right)^{-2}\right),
$$

so that

$$
\begin{aligned}
&\left\|\psi_{j}(\xi)\left(P(k ; \xi)-R_{\infty}^{n}\right)\right\|_{L_{2}}\left\|\frac{d}{d \xi}\left(\psi_{j}(\xi)\left(P(k ; \xi)-R_{\infty}^{n}\right)\right)\right\|_{L_{2}} \\
& \leq C \sum_{l=1}^{n}\left(1+\kappa_{l} 2^{j}\right)^{-1}, \quad j \geq 1 .
\end{aligned}
$$

Similarly, we derive as well

$$
\left\|\psi_{0}(\xi)\left(P(k ; \xi)-R_{\infty}^{n}\right)\right\|_{L_{2}} \leq C,
$$

and

$$
\begin{array}{r}
\left\|\frac{d}{d \xi}\left(\psi_{0}(\xi)\left(P(k ; \xi)-R_{\infty}^{n}\right)\right)\right\|_{L_{2}} \leq C\left(1+\sum_{l=1}^{n} \kappa_{l}\left(1+\kappa_{l}\right)^{-1 / 2}\right) \\
\leq C\left(1+\sum_{l=1}^{n} \kappa_{l}^{1 / 2}\right) .
\end{array}
$$

It follows now from the inequality (92) and the estimates (20) $-(22)$ that

$$
\begin{aligned}
\llbracket \psi_{j}(\xi)\left(P(k ; \xi)-R_{\infty}^{n}\right) \rrbracket & \leq C\left(\sum_{l=1}^{n}\left(1+\kappa_{l} 2^{j}\right)^{-1}\right)^{1 / 2} \\
& \leq C 2^{-j \varepsilon / 2} k^{\varepsilon / 2}\left(S_{-\varepsilon}\right)^{-\varepsilon / 2}
\end{aligned}
$$

for any $j \geq 1$ and $\varepsilon \in(0,1]$, and

$$
\begin{aligned}
\llbracket \psi_{0}(\xi)\left(P(k ; \xi)-R_{\infty}^{n}\right) \rrbracket & \leq C\left(1+\sum_{l=1}^{n} \kappa_{l}^{1 / 2}\right)^{1 / 2} \\
& \leq C\left(1+k^{-1 / 4}\left(S_{1 / 2}\right)^{1 / 4}\right) .
\end{aligned}
$$

Combining (13), (23), and (24) thus yields for any $\varepsilon \in(0,1]$,

$$
\llbracket P(k ; \xi) \rrbracket \leq C\left(1+k^{-1 / 4}\left(S_{1 / 2}\right)^{1 / 4}+\varepsilon^{-1} k^{\varepsilon / 2}\left(S_{-\varepsilon}\right)^{-\varepsilon / 2}\right),
$$

whence after optimization in $k$ and taking into account (12), we get for all $\varepsilon \in(0,1]$,

$$
\begin{aligned}
\llbracket P(\xi) \rrbracket & \leq C\left(1+\varepsilon^{-1}\left(\frac{S_{1 / 2}}{S_{-\varepsilon}}\right)^{\varepsilon /(4 \varepsilon+2)}\right) \\
& =C\left(1+\varepsilon^{-1} n^{1 / 2}\left(\frac{M_{1 / 2}}{M_{-\varepsilon}}\right)^{\varepsilon /(4 \varepsilon+2)}\right) .
\end{aligned}
$$

Observe that, in view of (5), the first term in the parentheses on the right-hand side of (25) can be omitted. The desired estimate (11) thus obtains by combining this and (10).

On the basis of the estimate (11), one can obtain simpler estimates which are more convenient for analysis. 
Corollary 2.1. Under the conditions of Theorem 2.1, for any finite stepsize sequence $K_{n}=\left(k_{j}\right)_{j=1}^{n}$, we have

$$
\left\|R\left(K_{n} ; A\right)\right\| \leq C L \log (1+\kappa(n)) n^{1 / 2},
$$

where

$$
\kappa(n)=\max _{1 \leq l \leq n} k_{l} / \min _{1 \leq l \leq n} k_{l} .
$$

Proof. It follows immediately from (11) that

$$
\left\|R\left(K_{n} ; A\right)\right\| \leq C L \varepsilon^{-1} n^{1 / 2} \kappa(n)^{\varepsilon} \quad \text { for all } \varepsilon \in(0,1],
$$

whence setting $\varepsilon=1 / \log (1+\kappa(n))$ implies (26).

We call a family $\mathcal{T}$ of stepsize sequences quasiuniform, if with $\kappa(n)$ defined as above,

$$
\sup _{K_{n} \subset K} \kappa(n) \leq C \text { for any } K \in \mathcal{T} .
$$

Corollary 2.2. Under the conditions of Theorem 2.1, for any quasiuniform family $\mathcal{T}$ of stepsize sequences and any $K_{n} \subset K \in \mathcal{T}$, the following estimate holds

$$
\left\|R\left(K_{n} ; A\right)\right\| \leq C L n^{1 / 2} .
$$

Proof. This is a straightforward consequence of (26).

The only restriction on $R(z)$ used in the statement of Theorem 2.1 is hypothesis HR 1 which is actually the condition of $A$-stability if $R(z)$ is interpreted as the stability function of an approximation method. Now, in order to obtain sharper estimates, we make slightly stronger restrictions on $R(z)$.

Theorem 2.2. Assume that the operator $A$ and the function $R(z)$ satisfy HA1 with $\nu=0, L>0$ and $\mathbf{H R} 1-5$ with $\varphi=\pi / 2, p \geq 1$, and $s \geq p+1$. Then, for any finite stepsize sequence $K_{n}=\left(k_{j}\right)_{j=1}^{n}$, we have

$$
\left\|R\left(K_{n} ; A\right)\right\| \leq C L n^{(1-(p+1) / s) / 2} .
$$

Proof. In view of (10), it suffices to bound the quantity $\llbracket P(\xi) \rrbracket$. Let $H_{n}$ be the ordered sequence for $K_{n}$, let $\left(l_{j}\right)_{j=1}^{\mu}$ be the associated sequence of indices for $K_{n}$, and let $\psi(\xi)$ be a smooth cut-off function with supp $\psi \subseteq[-1,1]$ satisfying the restriction

$$
\psi(\xi)=1 \quad \text { for }|\xi| \leq \frac{1}{2}
$$

One can easily check that

$$
\left(1-\psi\left(h_{l_{j+1}} \xi\right)\right)\left(1-\psi\left(h_{l_{j}} \xi\right)\right)=1-\psi\left(h_{l_{j}} \xi\right), \quad j=1, \ldots, \mu-1 .
$$

This allows us to use the following identity

$$
1=\psi\left(h_{l_{\mu}} \xi\right)+\sum_{j=1}^{\mu-1}\left(1-\psi\left(h_{l_{j+1}} \xi\right)\right) \psi\left(h_{l_{j}} \xi\right)+\left(1-\psi\left(h_{l_{1}} \xi\right)\right), \quad \xi \in \mathbb{R} .
$$

Further, recalling that $a(\xi)=e^{-i \xi} r(\xi)$, denote

$$
P_{j}(\xi)=\prod_{q=l_{j}+1}^{n} r\left(h_{q} \xi\right) \quad \text { and } \quad Q_{j}(\xi)=\prod_{q=1}^{l_{j}} a\left(h_{q} \xi\right) .
$$


Noting that

$$
P(\xi)=\prod_{l=1}^{n} r\left(h_{l} \xi\right)
$$

by the triangle inequality, (7), (30), and Lemma 6 in [4], we get

$$
\begin{aligned}
\llbracket P(\xi) \rrbracket \leq \llbracket \psi & \left(h_{l_{\mu}} \xi\right) Q_{\mu}(\xi) \rrbracket+\sum_{j=1}^{\mu-1} \llbracket\left(1-\psi\left(h_{l_{j+1}} \xi\right)\right) P_{j}(\xi) \rrbracket \\
& \times \llbracket \psi\left(h_{l_{j}} \xi\right) Q_{j}(\xi) \rrbracket+\llbracket\left(1-\psi\left(h_{l_{1}} \xi\right)\right) P(\xi) \rrbracket .
\end{aligned}
$$

Our next argument will be essentially based on the following two lemmas:

Lemma 2.1. Under the conditions of Theorem 2.2, for any finite stepsize sequence $K_{n}=\left(k_{j}\right)_{j=1}^{n}$ such that $k_{1} \leq \ldots \leq k_{n}$, we have

$$
\llbracket \psi\left(k_{n} \xi\right) \prod_{j=1}^{n} a\left(k_{j} \xi\right) \rrbracket \leq C\left(1+\left(\frac{S_{p+1}\left(K_{n}\right)}{S_{s}\left(K_{n}\right)}\right)^{(p+1) / 2}\right) .
$$

Lemma 2.2. Let $K_{n}=\left(k_{j}\right)_{j=1}^{n}$ be a finite stepsize sequence satisfying $k_{1} \leq \ldots \leq$ $k_{n}$ and let $1 \leq \sigma \leq n$ be an index such that $k_{\sigma} \leq 2 k_{1}$. Then, under the conditions of Theorem 2.2, we have

$$
\llbracket\left(1-\psi\left(k_{\sigma} \xi\right)\right) P\left(K_{n} ; \xi\right) \rrbracket \leq C \delta^{n},
$$

with some fixed $\delta \in(0,1)$.

The proofs of both lemmas will be given below.

Now we are ready to complete the proof of Theorem 2.2. Applying Lemmas 2.1 and 2.2 to (31), we find

$$
\llbracket P(\xi) \rrbracket \leq C\left(1+\sum_{j=1}^{\mu} \delta^{l_{\mu}-l_{j}}\left(\frac{S_{j, p+1}}{S_{j, s}}\right)^{(p+1) / 2}\right) .
$$

Note that, in view of (5) and since $p+1 \leq s$, we have

$$
\frac{S_{j, p+1}}{S_{j, s}}=l_{j}^{1 /(p+1)-1 / s} \frac{M_{j, p+1}}{M_{j, s}} \leq l_{j}^{1 /(p+1)-1 / s} \leq n^{1 /(p+1)-1 / s}, \quad j=1, \ldots, \mu,
$$

which yields instead of (34),

$$
\llbracket P(\xi) \rrbracket \leq C\left(1+n^{(1-(p+1) / s) / 2} \sum_{j=1}^{\mu} \delta^{n-l_{j}}\right) \leq C n^{(1-(p+1) / s) / 2}
$$

So (29) follows by combining (10) and (36).

It remains to prove Lemmas 2.1 and 2.2 .

Proof of Lemma 2.1. First of all denote

$$
Q(\xi)=\prod_{l=1}^{n} a\left(\frac{k_{l}}{k_{n}} \xi\right)
$$

Lemma 6 in 4 shows

$$
\llbracket \psi\left(k_{n} \xi\right) \prod_{l=1}^{n} a\left(k_{l} \xi\right) \rrbracket=\llbracket \psi(\xi) Q(\xi) \rrbracket .
$$


It is convenient to denote as well

$$
\Theta_{\beta}=k_{n}^{-1} S_{\beta}, \quad \beta>0 .
$$

Clearly, by the assumed restrictions on $k_{1}, \ldots, k_{n}$ and hypothesis HR5, we have

$$
|\psi(\xi) Q(\xi)| \leq C \exp \left(-c \Theta_{s}^{s}|\xi|^{s}\right) .
$$

Next, since

$$
a(\xi)=e^{\vartheta(\xi)} \quad \text { with } \quad \vartheta(\xi)=\mathcal{O}\left(|\xi|^{p+1}\right),
$$

by the same reasonings and $\mathbf{H R} 4$, we find

$$
\left|\frac{d}{d \xi}(\psi(\xi) Q(\xi))\right| \leq C \exp \left(-c \Theta_{s}^{s}|\xi|^{s}\right)\left(1+\Theta_{p+1}^{p+1}|\xi|^{p}\right) .
$$

Now it follows from (38) and (39) that

and

$$
\|\psi(\xi) Q(\xi)\|_{L_{2}} \leq C \Theta_{s}^{-1 / 2},
$$

$$
\left\|\frac{d}{d \xi}(\psi(\xi) Q(\xi))\right\|_{L_{2}} \leq C\left(\Theta_{s}^{-1 / 2}+\Theta_{s}^{1 / 2}\left(\frac{S_{p+1}}{S_{s}}\right)^{p+1}\right) .
$$

Therefore, since $\Theta_{s} \geq 1$, by virtue of (40), (41), and (9), we conclude

$$
\llbracket \psi(\xi) Q(\xi) \rrbracket \leq C\left(\Theta_{s}^{-1}+\left(\frac{S_{p+1}}{S_{s}}\right)^{p+1}\right)^{1 / 2} \leq C\left(1+\left(\frac{S_{p+1}}{S_{s}}\right)^{(p+1) / 2}\right),
$$

which, in view of (37), directly leads to (32).

Proof of Lemma 2.2. First denote $\kappa_{j}=k_{j} / k_{\sigma}$ and

$$
P_{j}(\xi)=\prod_{l=1}^{j} r\left(\kappa_{l} \xi\right)
$$

Next observe that, by Lemma 6 in $[4$,

$$
\llbracket\left(1-\psi\left(k_{\sigma} \xi\right)\right) \prod_{l=1}^{n} r\left(k_{l} \xi\right) \rrbracket=\llbracket(1-\psi(\xi)) P_{n}(\xi) \rrbracket .
$$

Further, by the identity

$$
P_{n}(\xi)=\sum_{j=1}^{n} R_{\infty}^{n-j}\left(r\left(\kappa_{j} \xi\right)-R_{\infty}\right) P_{j-1}(\xi)+R_{\infty}^{n},
$$

and the triangle inequality, we obtain

$$
\begin{aligned}
\llbracket(1-\psi(\xi)) P_{n}(\xi) \rrbracket \leq & \left|R_{\infty}\right|^{n}+\sum_{j=1}^{n}\left|R_{\infty}\right|^{n-j} \\
& \times \llbracket(1-\psi(\xi))\left(r\left(\kappa_{j} \xi\right)-R_{\infty}\right) P_{j-1}(\xi) \rrbracket .
\end{aligned}
$$

Note that by our assumptions,

$$
\kappa_{j} \geq \frac{1}{2}, \quad j=1, \ldots, n,
$$


and, in view of $\mathbf{H R 2}$ and $\mathbf{H R} 3$, there exists $\delta_{1} \in(0,1)$ such that

$$
|r(\xi)| \leq \delta_{1} \quad \text { for }|\xi| \geq \frac{1}{4}
$$

Using (15), (44), and (45), we get

$$
\left|(1-\psi(\xi))\left(r\left(\kappa_{j} \xi\right)-R_{\infty}\right) P_{j-1}(\xi)\right| \leq C \delta_{1}^{j}\left(1+\kappa_{j}|\xi|\right)^{-1} .
$$

Applying the same argument together with (16) yields

$$
\begin{aligned}
& \mid \frac{d}{d \xi}((1-\psi(\xi))\left.\left(r\left(\kappa_{j} \xi\right)-R_{\infty}\right) P_{j-1}(\xi)\right) \mid \\
& \leq C \delta_{1}^{j}\left(\left(1+\kappa_{j}|\xi|\right)^{-1}+\sum_{l=1}^{j} \kappa_{l}\left(1+\kappa_{l}|\xi|\right)^{-2}\right) .
\end{aligned}
$$

On the basis of (46) and (47), we derive

$$
\left\|(1-\psi(\xi))\left(r\left(\kappa_{j} \xi\right)-R_{\infty}\right) P_{j-1}(\xi)\right\|_{L_{2}} \leq C \delta_{1}^{j} \kappa_{j}^{-1 / 2}
$$

and

$$
\begin{aligned}
& \| \frac{d}{d \xi}\left((1-\psi(\xi))\left(r\left(\kappa_{j} \xi\right)-R_{\infty}\right) P_{j-1}(\xi)\right) \|_{L_{2}} \\
& \leq C \delta_{1}^{j}\left(\kappa_{j}^{-1 / 2}+\sum_{l=1}^{j} \kappa_{l}^{1 / 2}\right) \leq C \delta_{1}^{j}\left(\kappa_{j}^{-1 / 2}+j \kappa_{j}^{1 / 2}\right) .
\end{aligned}
$$

Using (48), (49), and (9), and taking into account (44), we find

$$
\llbracket(1-\psi(\xi))\left(r\left(\kappa_{j} \xi\right)-R_{\infty}\right) P_{j-1}(\xi) \rrbracket \leq C \delta_{1}^{j}\left(\kappa_{j}^{-1}+j\right)^{1 / 2} \leq C j^{1 / 2} \delta_{1}^{j} .
$$

Inserting (50) into (43) yields

$$
\llbracket(1-\psi(\xi)) P_{n}(\xi) \rrbracket \leq C \sum_{j=1}^{n} j^{1 / 2} \delta_{1}^{j}\left|R_{\infty}\right|^{n-j}+\left|R_{\infty}\right|^{n} \leq C \delta^{n},
$$

with some $\delta \in\left(\delta_{1}, 1\right)$, whence (33) obtains in view of (42).

\section{Semigroups With EXPONENTIAL DECAY}

In this section, we study asymptotic properties of rational approximations to $C_{0}$ semigroups with exponential decay. In other words, we assume that hypothesis HA1 is fulfilled with $\nu>0$.

For our further needs, it is convenient to introduce the shifted operator

$$
B=A+\nu I
$$

This operator generates a bounded semigroup $e^{t B}$ satisfying

$$
\left\|e^{t B}\right\| \leq L \text { for all } t \geq 0
$$

Therefore, the operator $B$ fulfils hypothesis HA1 with $\nu=0$ and one can use the Hille-Phillips operator calculus in terms of $B$. Assume that the function $R(z)$ satisfies HR1 with $\varphi=\pi / 2$. Using the same reasonings that lead to (10) for the operator $B$ instead of $A$, we obtain for any finite stepsize sequence $K_{n}=\left(k_{j}\right)_{j=1}^{n}$,

$$
\left\|R\left(K_{n} ; A\right)\right\|=\left\|R\left(K_{n} ; B-\nu I\right)\right\| \leq L \llbracket \prod_{l=1}^{n} r\left(k_{l} \xi,-k_{l} \nu\right) \rrbracket .
$$


The last inequality will be of essential use in the sequel.

Now we can state our results on asymptotic stability.

Theorem 3.1. Let the operator $A$ and the function $R(z)$ satisfy the hypotheses HA1 with $L>0, \nu>0$, and HR $1-5$ with $\varphi=\pi / 2$ and $p \geq 1, s \geq p+1$. Let further $\mathcal{T}$ be the set of stepsize sequences satisfying the restriction

$$
k_{j} \leq \kappa, \quad j=1,2, \ldots \quad \text { for any }\left(k_{j}\right)_{j=1}^{\infty} \in \mathcal{T},
$$

with some fixed $\kappa>0$. Then, if $\kappa \nu>0$ is sufficiently small, there exists $\omega \in(0, \nu]$ such that for any $K_{n} \subset K \in \mathcal{T}$,

$$
\left\|R\left(K_{n} ; A\right)\right\| \leq C L e^{-\omega t_{n}} n^{(1-(p+1) / s) / 2} .
$$

Moreover, for $\kappa>0$ sufficiently small, $\omega$ can be chosen as close to $\nu$ as desired.

Proof. In view of 52, it suffices to bound the quantity

$$
\llbracket \prod_{j=1}^{n} r\left(k_{j} \xi,-k_{j} \nu\right) \rrbracket
$$

Let $H_{n}=\left(h_{j}\right)_{j=1}^{n}$ be the ordered sequence for $K_{n}$ and let $\left(l_{j}\right)_{j=1}^{\mu}$ be the associated sequence of indices for $K_{n}$. As above, when bounding (54), the finite sequence $K_{n}=\left(k_{j}\right)_{j=1}^{n}$ in (54) can be replaced by $H_{n}$.

Next let $\psi(\xi)$ be a smooth cut-off function such that for some fixed $0<\rho \leq 1$,

$$
\operatorname{supp} \psi \subseteq[-\rho, \rho] \quad \text { and } \quad \psi(\xi)=1 \quad \text { for }|\xi| \leq \frac{\rho}{2} .
$$

Below we shall use some reasonings applied in the proof of Theorem 2.2, where we introduced $\psi(\xi)$ in just the same way as above but with $\rho=1$.

Further, denote

$$
a(\xi, \eta)=e^{-i \xi} r(\xi, \eta)
$$

Similarly to (31), we have

$$
\begin{aligned}
\llbracket \prod_{l=1}^{n} r\left(h_{l} \xi,-h_{l} \nu\right) \rrbracket \leq & \llbracket \psi\left(h_{l_{\mu}} \xi\right) \prod_{l=1}^{n} a\left(h_{l} \xi,-h_{l} \nu\right) \rrbracket \\
& +\sum_{j=1}^{\mu-1} \llbracket\left(1-\psi\left(h_{l_{j+1}} \xi\right)\right) \prod_{l=l_{j}+1}^{n} r\left(h_{l} \xi,-h_{l} \nu\right) \rrbracket \\
& \times \llbracket \psi\left(h_{l_{j}} \xi\right) \prod_{l=1}^{l_{j}} a\left(h_{l} \xi,-h_{l} \nu\right) \rrbracket \\
& +\llbracket\left(1-\psi\left(h_{l_{1}} \xi\right)\right) \prod_{l=1}^{n} r\left(h_{l} \xi,-h_{l} \nu\right) \rrbracket .
\end{aligned}
$$

Using the same argument as in the proof of Lemma 2.2, we obtain (taking $l_{0}=0$ )

$$
\llbracket\left(1-\psi\left(h_{l_{j+1}} \xi\right)\right) \prod_{l=l_{j}+1}^{n} r\left(h_{l} \xi,-h_{l} \nu\right) \rrbracket \leq C \delta^{n-l_{j}}, \quad j=0, \ldots, \mu-1,
$$

with some fixed $\delta \in(0,1)$. 
In order to bound the quantities

$$
\llbracket \psi\left(h_{l_{j}} \xi\right) \prod_{l=1}^{l_{j}} a\left(h_{l} \xi,-h_{l} \nu\right) \rrbracket,
$$

we denote $\kappa_{j, q}=h_{q} / h_{l_{j}}$ and

$$
Q_{j}(\xi)=\prod_{q=1}^{l_{j}} a\left(\kappa_{j, q} \xi,-h_{q} \nu\right)
$$

and use the identity (see Lemma 6 in Brenner and Thomée [4])

$$
\llbracket \psi\left(h_{l_{j}} \xi\right) \prod_{l=1}^{l_{j}} a\left(h_{l} \xi,-h_{l} \nu\right) \rrbracket=\llbracket \psi(\xi) Q_{j}(\xi) \rrbracket .
$$

Further, by HR4 - 5 we have

$$
R(z)=e^{z} e^{\vartheta(z)}
$$

where

$$
\vartheta(z)=\mathcal{O}\left(|z|^{p+1}\right) \quad \text { as } z \rightarrow 0
$$

and

$$
\operatorname{Re} \vartheta(i \xi) \leq-c \xi^{s} \text { for }|\xi| \leq \rho
$$

Using the expansion

$$
\vartheta\left(i \kappa_{j, q} \xi-h_{q} \nu\right)=\vartheta\left(i \kappa_{j, q} \xi\right)-h_{q} \nu \vartheta^{\prime}\left(i \kappa_{j, q} \xi-\zeta_{q}\right), \quad q=1, \ldots, l_{j},
$$

with some $\zeta_{q} \in\left(0, h_{q} \nu\right)$, and taking into account (59) and (60), we conclude that for all $|\xi| \leq \rho$,

$$
\begin{array}{r}
\left|\exp \left(\vartheta\left(i \kappa_{j, q} \xi-h_{q} \nu\right)\right)\right| \leq\left|\exp \left(\vartheta\left(i \kappa_{j, q} \xi\right)\right)\right| \exp \left(C h_{q} \nu\left|i \kappa_{j, q} \xi-\zeta_{q}\right|^{p}\right) \\
\leq \exp \left(-c \kappa_{j, q}^{s} \xi^{s}\right) \exp \left(C h_{q} \nu\left(\rho^{p}+(\kappa \nu)^{p}\right)\right), \quad q=1, \ldots, l_{j} .
\end{array}
$$

Therefore, choosing $\rho>0$ sufficiently small, we obtain by (158) and (61), with $\kappa \nu>0$ sufficiently small for all $|\xi| \leq \rho$,

$$
\begin{aligned}
\left|a\left(\kappa_{j, q} \xi,-h_{q} \nu\right)\right| & \leq \exp \left(-h_{q} \nu-c \kappa_{j, q}^{s} \xi^{s}+C h_{q} \nu\left(\rho^{p}+(\kappa \nu)^{p}\right)\right) \\
& \leq \exp \left(-h_{q} \omega_{1}-c \kappa_{j, q}^{s} \xi^{s}\right), \quad q=1, \ldots, l_{j},
\end{aligned}
$$

where $\omega_{1} \in(0, \nu)$ can be chosen as close to $\nu$ as desired. Applying (591), (61), and the above argument, we derive as well, with $\rho>0$ and $\kappa \nu>0$ sufficiently small, for all $|\xi| \leq \rho$,

$$
\begin{aligned}
& \left|\frac{d}{d \xi} a\left(\kappa_{j, q} \xi,-h_{q} \nu\right)\right| \leq \exp \left(-h_{q} \omega_{1}-c \kappa_{j, q}^{s} \xi^{s}\right) \kappa_{j, q}\left|i \kappa_{j, q} \xi-h_{q} \nu\right|^{p} \\
& \quad \leq \exp \left(-h_{q} \omega_{1}-c \kappa_{j, q}^{s} \xi^{s}\right)\left(\kappa_{j, q}^{p+1}|\xi|^{p}+\kappa_{j, q}\left(h_{q} \nu\right)^{p}\right), \quad q=1, \ldots, l_{j},
\end{aligned}
$$

where $\omega_{1}$ is just the same as in (62). Denoting

$$
\Theta_{j, \beta}=\frac{S_{j, \beta}}{h_{l_{j}}}, \quad \beta>0,
$$

and using (62) and (63), we find

$$
\left|\psi(\xi) Q_{j}(\xi)\right| \leq \exp \left(-S_{j, 1} \omega_{1}-c \Theta_{j, s}^{s} \xi^{s}\right),
$$


and

$$
\begin{aligned}
\left|\frac{d}{d \xi}\left(\psi(\xi) Q_{j}(\xi)\right)\right| \leq & C \exp \left(-S_{j, 1} \omega_{1}-c \Theta_{j, s}^{s} \xi^{s}\right) \\
& +C \exp \left(-S_{j, 1} \omega_{1}-c \Theta_{j, s}^{s} \xi^{s}\right)\left(\Theta_{j, p+1}^{p+1}|\xi|^{p}+S_{j, p+1}^{p+1} \frac{\nu^{p}}{h_{l_{j}}}\right) .
\end{aligned}
$$

It follows from (64) and (65) that

$$
\left\|\psi(\xi) Q_{j}(\xi)\right\|_{L_{2}} \leq C e^{-S_{j, 1} \omega_{1}} \Theta_{j, s}^{-1 / 2},
$$

and

$$
\begin{aligned}
\left\|\frac{d}{d \xi}\left(\psi(\xi) Q_{j}(\xi)\right)\right\|_{L_{2}} & \leq C e^{-S_{j, 1} \omega_{1}} \Theta_{j, s}^{-1 / 2}\left(1+\frac{S_{j, p+1}^{p+1}}{h_{l_{j}}} \nu^{p}\right) \\
& +C e^{-S_{j, 1} \omega_{1}}\left(\frac{S_{j, p+1}}{S_{j, s}}\right)^{p+1} \Theta_{j, s}^{1 / 2} .
\end{aligned}
$$

Since $\Theta_{j, s} \geq 1$, we get by (97), (66), and (67),

$$
\begin{aligned}
\llbracket \psi(\xi) Q_{j}(\xi) \rrbracket & \leq C e^{-S_{j, 1} \omega_{1}}\left(\Theta_{j, s}^{-1}\left(1+\frac{S_{j, p+1}^{p+1}}{h_{l_{j}}} \nu^{p}\right)+\left(\frac{S_{j, p+1}}{S_{j, s}}\right)^{p+1}\right)^{1 / 2} \\
& \leq C e^{-S_{j, 1} \omega_{1}}\left(1+\frac{S_{j, p+1}^{p+1}}{S_{j, s}}+\left(\frac{S_{j, p+1}}{S_{j, s}}\right)^{p+1}\right)^{1 / 2} .
\end{aligned}
$$

In view of (4), we have

$$
S_{j, s} \leq S_{j, p+1} \leq S_{j, 1}
$$

This yields

$$
\begin{aligned}
\llbracket \psi(\xi) Q_{j}(\xi) \rrbracket & \leq C e^{-S_{j, 1} \omega_{1}}\left(1+S_{j, 1}^{p} \frac{S_{j, p+1}}{S_{j, s}}+\left(\frac{S_{j, p+1}}{S_{j, s}}\right)^{p+1}\right)^{1 / 2} \\
& \leq C e^{-S_{j, 1} \omega_{1}}\left(1+S_{j, 1}^{p}\right)^{1 / 2}\left(\frac{S_{j, p+1}}{S_{j, s}}\right)^{(p+1) / 2} \\
& \leq C e^{-S_{j, 1} \omega}\left(\frac{S_{j, p+1}}{S_{j, s}}\right)^{(p+1) / 2}
\end{aligned}
$$

where $\omega \in\left(0, \omega_{1}\right)$ can be chosen as close to $\omega_{1}$, and hence to $\nu$, as desired. It is convenient to denote

$$
m=(1-(p+1) / s) / 2 .
$$

Together, (699) and (35) yield

$$
\llbracket \psi(\xi) Q_{j}(\xi) \rrbracket \leq C e^{-S_{j, 1} \omega} n^{m}, \quad j=1, \ldots, \mu .
$$

Therefore, by combining (55)), (56), (57), and (70), we get

$$
\llbracket \prod_{l=1}^{n} r\left(h_{l} \xi,-h_{l} \nu\right) \rrbracket \leq C n^{m} \sum_{j=1}^{\mu} e^{-S_{j, 1} \omega} \delta^{n-l_{j}}+C \delta^{n} .
$$

Select some $\delta_{1} \in(\delta, 1)$. Clearly, for $\kappa \nu>0$ sufficiently small, we have

$$
\delta_{1}^{n-l_{j}} \leq e^{-\kappa \nu\left(l_{\mu}-l_{j}\right)} \leq e^{-\nu\left(t_{n}-S_{j, 1}\right)} \leq e^{-\omega\left(t_{n}-S_{j, 1}\right)}, \quad j=1, \ldots, \mu,
$$


and thus

$$
\sum_{j=1}^{\mu} e^{-S_{j, 1} \omega} \delta^{n-l_{j}} \leq \sum_{j=1}^{\mu} e^{-S_{j, 1} \omega} \delta_{1}^{n-l_{j}}\left(\frac{\delta}{\delta_{1}}\right)^{n-l_{j}} \leq e^{-\omega t_{n}}
$$

Since

$$
\delta^{n} \leq e^{-\kappa n \nu} \leq e^{-\omega t_{n}},
$$

it follows from (71) and (73) that

$$
\llbracket \prod_{j=1}^{n} r\left(h_{j} \xi,-h_{j} \nu\right) \rrbracket \leq C n^{m} e^{-\omega t_{n}},
$$

which concludes the proof.

If the stepsize sequence is not uniformly bounded, an exponential factor as in (53) cannot be expected. This is easily seen by an example: take $A=-1$ and consider the backward Euler approximations with stepsizes $k_{n}=2^{n}$. In this situation, an exponential bound with positive $\omega$ does not hold.

In order to show algebraic decay, we formulate the following condition on the family of stepsize sequences $\mathcal{T}$ :

HS1: There exists $Q>1$ such that for any $K_{n} \subset K \in \mathcal{T}$,

$$
t_{n} \leq C Q^{n}
$$

and

$$
t_{n} \leq C Q^{n-j} \sum_{l=1}^{j} h_{l}, \quad j=1, \ldots, n,
$$

where $H_{n}=\left(h_{j}\right)_{j=1}^{n}$ is the ordered sequence for $K_{n}$.

Example. Let $Q>1$ and let $\mathcal{T}$ be a set of stepsize sequences satisfying

$$
k_{n} \leq k_{n+1} \leq Q k_{n}, \quad n=1,2, \ldots, \text { and } k_{1} \leq C .
$$

Then, $\mathcal{T}$ satisfies HS1 with a constant $C$ that depends on $Q$ and on the bound of the first step $k_{1}$. This follows from

$$
t_{n}=t_{j}+\sum_{l=j+1}^{n} k_{l} \leq\left(1+\frac{Q}{Q-1}\left(Q^{n-j}-1\right)\right) t_{j} \leq \frac{Q}{Q-1} Q^{n-j} t_{j},
$$

which shows (76), as well as from

$$
t_{n} \leq k_{1} \sum_{l=0}^{n-1} Q^{l} \leq \frac{k_{1}}{Q-1} Q^{n}
$$

which implies (75).

Theorem 3.2. Let the operator $A$ and the function $R(z)$ satisfy the hypotheses HA 1 with $\nu>0$ and $L>0$, and $\mathbf{H R} 1-5$ with $\varphi=\pi / 2$ and $p \geq 1, s \geq p+1$. Let $\mathcal{T}$ be a set of stepsize sequences satisfying $\mathbf{H S} 1$ and let $\alpha>0$ be such that

$$
Q^{\alpha} \in\left(1,\left|R_{\infty}\right|^{-1}\right) \text {. }
$$

Then, for any $K_{n} \subset K \in \mathcal{T}$, we have the estimate

$$
\left\|R\left(K_{n} ; A\right)\right\| \leq C L n^{(1-(p+1) / s) / 2}\left(1+t_{n}^{\alpha}\right)^{-1} .
$$


Note that for methods with $R_{\infty}=0$, e.g., for the Radau methods, the value of $\alpha$ is not restricted by (77) and can be chosen as large as desired.

The proof of this theorem is based on the following lemma:

Lemma 3.1. Assume that the function $R(z)$ satisfies the conditions of Theorem 3.2. Let $K_{n}$ be any finite stepsize sequence such that

$$
\nu \min \left(k_{1}, \ldots, k_{n}\right) \geq \gamma_{0}>0 .
$$

Then

$$
\llbracket \prod_{j=1}^{n} r\left(k_{j} \xi,-k_{j} \nu\right) \rrbracket \leq C n^{3 / 2} \delta_{0}^{n}
$$

where

$$
\delta_{0}=\sup _{\operatorname{Re} z \leq-\gamma_{0}}|R(z)|<1
$$

Proof. It suffices to prove the lemma with $H_{n}$ substituted for $K_{n}$, where $H_{n}=$ $\left(h_{j}\right)_{j=1}^{n}$ is the ordered sequence for $K_{n}$. We fix $1 \leq j \leq n$ and denote

$$
r_{j}(\xi)=r\left(h_{j} \xi,-h_{j} \nu\right)-R_{\infty},
$$

and

$$
P_{j}(\xi)=\prod_{l=1}^{j} r\left(h_{l} \xi,-h_{l} \nu\right)
$$

Under the assumed restrictions, we have

$$
\left|r\left(h_{j} \xi,-h_{j} \nu\right)\right| \leq \delta_{0} \quad \text { for all } \xi \in \mathbb{R} .
$$

Next, using the estimate (79) and the inequalities

$$
\left|r_{j}(\xi)\right| \leq C \min \left(\delta_{0},\left(1+h_{j}|\xi|\right)^{-1}\right),
$$

and

$$
\left|\frac{d}{d \xi} r_{j}(\xi)\right| \leq C h_{j}\left(1+h_{j}|\xi|\right)^{-1}
$$

we get

$$
\left|r_{j}(\xi) P_{j-1}(\xi)\right| \leq C\left(1+h_{j}|\xi|\right)^{-1} \delta_{0}^{j}
$$

and

$$
\left|\frac{d}{d \xi}\left(r_{j}(\xi) P_{j-1}(\xi)\right)\right| \leq C \delta_{0}^{j} \sum_{q=1}^{j} h_{q}\left(1+h_{q}|\xi|\right)^{-1} .
$$

It follows from (80) and (81) that

$$
\left\|r_{j}(\xi) P_{j-1}(\xi)\right\|_{L_{2}} \leq C h_{j}^{-\frac{1}{2}} \delta_{0}^{j},
$$

and

$$
\left\|\frac{d}{d \xi}\left(r_{j}(\xi) P_{j-1}(\xi)\right)\right\|_{L_{2}} \leq C \delta_{0}^{j} \sum_{q=1}^{j} h_{q}^{\frac{1}{2}}
$$


whence by (9),

$$
\llbracket r_{j}(\xi) P_{j-1}(\xi) \rrbracket \leq C \delta_{0}^{j}\left(\sum_{q=1}^{j}\left(\frac{h_{q}}{h_{j}}\right)^{\frac{1}{2}}\right)^{1 / 2} \leq C j^{1 / 2} \delta_{0}^{j} .
$$

On the basis of the last estimate, we find

$$
\begin{aligned}
\llbracket \prod_{l=1}^{n} r\left(h_{l} \xi,-h_{l} \nu\right) \rrbracket & \leq \sum_{j=1}^{n}\left|R_{\infty}\right|^{n-j} \llbracket r_{j}(\xi) P_{j-1}(\xi) \rrbracket+\llbracket R_{\infty}^{n} \rrbracket \\
& \leq C \sum_{j=1}^{n} j^{1 / 2} \delta_{0}^{j}\left|R_{\infty}\right|^{n-j}+\left|R_{\infty}\right|^{n} \leq C n^{3 / 2} \delta_{0}^{n},
\end{aligned}
$$

which concludes the proof.

Proof of Theorem 3.2. Let $K \in \mathcal{T}$ and let $K_{n} \subset K$. It suffices to show the result for the ordered sequence $H_{n}=\left(h_{j}\right)_{j=1}^{n}$ substituted for $K_{n}$.

Let $\delta_{0}$ be any fixed number such that $\delta_{0} \in\left(\left|R_{\infty}\right|, Q^{-\alpha}\right)$, with $Q$ given in HS1. Given $n$, let $j_{n}$ be the index such that

$$
h_{j} \nu<\gamma_{0}, \quad j=1, \ldots, j_{n},
$$

and

$$
h_{j} \nu \geq \gamma_{0}, \quad j=j_{n}+1, \ldots, n,
$$

where $\gamma_{0}>0$ is chosen such that

$$
\sup _{\operatorname{Re} z \leq-\gamma_{0}}|R(z)|=\delta_{0} .
$$

We therefore have

$$
\llbracket \prod_{j=1}^{n} r\left(h_{j} \xi,-h_{j} \nu\right) \rrbracket \leq \llbracket \prod_{j=1}^{j_{n}} r\left(h_{j} \xi,-h_{j} \nu\right) \rrbracket \llbracket \prod_{j=j_{n}+1}^{n} r\left(h_{j} \xi,-h_{j} \nu\right) \rrbracket .
$$

By (84), (85), and Lemma 3.1 we find

$$
\llbracket \prod_{j=j_{n}+1}^{n} r\left(h_{j} \xi,-h_{j} \nu\right) \rrbracket \leq C\left(n-j_{n}\right)^{3 / 2} \delta_{0}^{n-j_{n}} .
$$

In order to bound the first factor on the right-hand side of (86), we assume for a moment that $\gamma_{0}>0$ is sufficiently small. If this is the case, the argument leading to the estimate (74) yields with some $\omega>0$,

$$
\llbracket \prod_{j=1}^{j_{n}} r\left(h_{j} \xi,-h_{j} \nu\right) \rrbracket \leq C j_{n}^{m} e^{-\omega \tau_{j_{n}}}
$$

where $m=(1-(p+1) / s) / 2$ and $\tau_{j_{n}}=\sum_{j=1}^{j_{n}} h_{j}$. If $\gamma_{0}$ is not sufficiently small, noting that both factors on the right-hand side of (86) are estimated independently, and reducing, if necessary, $\nu>0$ in HA1, we can nevertheless, for the estimation of the first factor, consider $h_{j} \nu>0, j=1, \ldots, j_{n}$, to be sufficiently small. This means that (88) is valid for any $\gamma_{0}>0$. Combining (52), (86), (87), and (88) thus yields

$$
\left\|R\left(H_{n} ; A\right)\right\| \leq C L\left(n-j_{n}\right)^{3 / 2} \delta_{0}^{n-j_{n}} j_{n}^{m} e^{-\omega \tau_{j_{n}}},
$$


and since $\delta_{0} Q^{\alpha}<1$,

$$
\begin{aligned}
\left\|R\left(H_{n} ; A\right)\right\| & \leq C L\left(n-j_{n}\right)^{3 / 2}\left(\delta_{0} Q^{\alpha}\right)^{n-j_{n}} Q^{-\left(n-j_{n}\right) \alpha} n^{m} e^{-\omega \tau_{j_{n}}} \\
& \leq C L Q^{-\left(n-j_{n}\right) \alpha} n^{m} e^{-\omega \tau_{j_{n}}} .
\end{aligned}
$$

Finally, in view of (76) and (75),

$$
Q^{-\left(n-j_{n}\right) \alpha} \leq C\left(1+\tau_{j_{n}}^{\alpha}\right)\left(1+t_{n}^{\alpha}\right)^{-1}
$$

and it follows from (90) that

$$
\left\|R\left(H_{n} ; A\right)\right\| \leq C L n^{m}\left(1+t_{n}^{\alpha}\right)^{-1}\left(1+\tau_{j_{n}}^{\alpha}\right) e^{-\omega \tau_{j_{n}}} \leq C L n^{m}\left(1+t_{n}^{\alpha}\right)^{-1},
$$

which concludes the proof.

Theorem 3.3. Let the operator $A$ and the function $R(z)$ fulfil the hypotheses HA1 with some $L>0$ and $\nu>0$, and $\mathbf{H R} 1-5$ with $\varphi=\pi / 2$ and $s=p+1$. Then, for any stepsize sequence $K=\left(k_{j}\right)_{j=1}^{\infty}$ such that $t_{n} \rightarrow \infty$ as $n \rightarrow \infty$, we have

$$
\left\|R\left(K_{n} ; A\right)\right\| \rightarrow 0 \quad \text { as } n \rightarrow \infty .
$$

Proof. Note that formula (89) is proved without any restrictions on $K=\left(k_{j}\right)_{j=1}^{\infty}$. So far as $s=p+1$, (89) implies with some $\delta_{0} \in(0,1)$ and $\omega>0$, for any finite sequence $K_{n}$,

$$
\left\|R\left(K_{n} ; A\right)\right\| \leq C L\left(n-j_{n}\right) \delta_{0}^{n-j_{n}} e^{-\omega \tau_{j_{n}}},
$$

where $j_{n}$ and $\tau_{j_{n}}$ are just the same as in the proof of Theorem 3.2

We have only two possibilities: either $\tau_{j_{n}}$ tends to $\infty$ or $\tau_{j_{n}}$ remains bounded. In the first case, (92) straightforwardly implies (911). We thus concentrate on the second case. By the definition of $j_{n}$, the assumption that $n-j_{n} \leq C$ yields

$$
t_{n}-\tau_{j_{n}} \leq C
$$

and since $t_{n}$ tends to infinity, we come to a contradiction. Therefore,

$$
\lim _{n \rightarrow \infty}\left(n-j_{n}\right)=\infty
$$

and using (92) again immediately leads to (91).

\section{HolOMORPHIC SEMigRoups}

The factor $n^{(1-(p+1) / s) / 2}$, appearing in the estimates (53) and (78), plays a harmful role in the asymptotic behavior of discretizations. It is absent only in the case $s=p+1$, which is a severe restriction on the possible choice of discretizations. A noteworthy exception satisfying $s=p+1$, however, are the Radau methods, i.e., the first subdiagonal Padé approximations to the exponential function.

In this section, we show that approximations to holomorphic semigroups possess better asymptotic properties in the sense that no factor, growing with $n$, appears in stability estimates. Note that our techniques require $\left|R_{\infty}\right|<1$, which excludes, for example, the Gauss methods.

Theorem 4.1. Let the operator $A$ and the function $R(z)$ fulfil the hypotheses HA2 with $\nu>0, L>0, \chi \in(0, \pi / 2)$ and HR1, HR2, HR4 with $\varphi \geq \chi$, and $p \geq 1$. Let further $\mathcal{T}$ be the set of stepsize sequences satisfying the restriction

$$
k_{j} \leq \kappa, \quad j=1,2, \ldots \quad \text { for any }\left(k_{j}\right)_{j=1}^{\infty} \in \mathcal{T}
$$


with some fixed $\kappa>0$. Then, if $\kappa \nu>0$ is sufficiently small, there exists an $\omega \in(0, \nu]$ such that for any $K_{n} \subset K \in \mathcal{T}$,

$$
\left\|R\left(K_{n} ; A\right)\right\| \leq C L e^{-\omega t_{n}} .
$$

Moreover $\omega$ can be chosen such that $\omega \geq \nu\left(1-c \cdot(\kappa \nu)^{p}\right)$, with $c>0$.

Proof. Our argument is close to that applied in the proof of Lemma 3.1 in [3]. Noting, as above, that it suffices to show (93) with $H_{n}$ substituted for $K_{n}$, where $H_{n}$ is the ordered sequence for $K_{n}$, we start with the identity

$$
R\left(H_{n} ; A\right)=\sum_{j=1}^{n} R_{\infty}^{n-j}\left(R\left(h_{j} A\right)-R_{\infty} I\right) \prod_{l=1}^{j-1} R\left(h_{l} A\right)+R_{\infty}^{n} I .
$$

Denote

$$
\tau_{j}=\sum_{l=1}^{j} h_{l}
$$

and assume for a moment that we have already shown the following estimate

$$
\left\|\left(R\left(h_{j} A\right)-R_{\infty} I\right) \prod_{l=1}^{j-1} R\left(h_{l} A\right)\right\| \leq C L e^{-\omega \tau_{j}}, \quad j=1, \ldots, n,
$$

with $\omega / \nu=1+\mathcal{O}\left((\kappa \nu)^{p}\right)$. Then (94) and (95) imply

$$
\left\|R\left(H_{n} ; A\right)\right\| \leq C L \sum_{j=1}^{n}\left|R_{\infty}\right|^{n-j} e^{-\omega \tau_{j}}+\left|R_{\infty}\right|^{n},
$$

and, for $\kappa \nu>0$ sufficiently small, the same reasoning as in the proof of Theorem 3.1 leads immediately to (93).

Therefore it remains to prove (95). In order to do this, we use the Dunford-Taylor operator calculus (see, e.g., Hille and Phillips [8]). We select constants $D \geq d>0$ with $d$ such that the disc $|z| \leq d$ contains no poles of $R(z)$.

Let the contour $\Gamma^{(j)}$ be given by

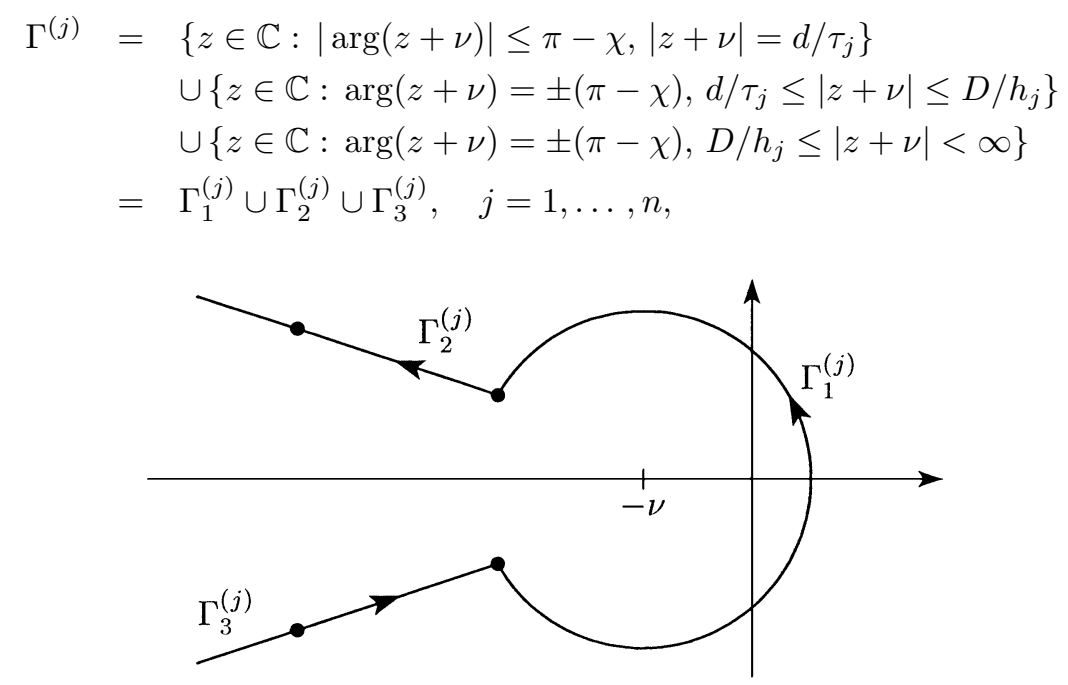

Figure 1. The used path of integration. 
and be oriented counter-clockwise, see Figure 1 For $q=1,2,3$, let

$$
I_{q}=\frac{1}{2 \pi i} \int_{\Gamma_{q}^{(j)}}\left(R\left(h_{j} z\right)-R_{\infty}\right) \prod_{l=1}^{j-1} R\left(h_{l} z\right)(z I-A)^{-1} d z .
$$

We have the representation

$$
\left(R\left(h_{j} A\right)-R_{\infty} I\right) \prod_{l=1}^{j-1} R\left(h_{l} A\right)=I_{1}+I_{2}+I_{3} .
$$

Note that by HR4,

$$
R(z)=e^{z} e^{\vartheta(z)} \text { with } \vartheta(z)=\mathcal{O}\left(|z|^{p+1}\right) \text { as } z \rightarrow 0 .
$$

It is seen from (98) that for $\kappa \nu>0$ and $d>0$ sufficiently small,

$$
\left|R\left(-h_{l} \nu+z\right)\right| \leq\left|e^{-h_{l} \nu+z}\right| e^{\left|\vartheta\left(-h_{l} \nu+z\right)\right|} \leq e^{-\omega h_{l}} e^{c|z|} \quad \text { for }|z| \leq d,
$$

with $\omega / \nu=1+\mathcal{O}\left((\kappa \nu)^{p}\right)$. With this in mind, we have by HA2,

$$
\left\|I_{1}\right\| \leq C L e^{-\omega \tau_{j}} \int_{\Gamma_{1}^{(j)}} e^{C|z+\nu| \tau_{j}}|z+\nu|^{-1}|d z| \leq C L e^{-\omega \tau_{j}} .
$$

Next, applying (98) once more, we find for $\kappa \nu$ and $D$ sufficiently small,

$$
\left|R\left(-h_{l} \nu+x e^{ \pm i(\pi-\chi)}\right)\right| \leq e^{-h_{l} \omega-c x}, \quad l=1, \ldots, j,
$$

for all $0 \leq x \leq D$, where $\omega / \nu=1+\mathcal{O}\left((\kappa \nu)^{p}\right)$. In view of HR2, however, (101) is valid with any fixed $D>0$ if $\kappa \nu>0$ is sufficiently small. Using this fact and the simple inequality

$$
1 \leq C e^{-h_{j} \omega-c h_{j} x} \quad \text { for } 0 \leq x \leq D / h_{j}
$$

we find by HA2,

$$
\left\|I_{2}\right\| \leq C L e^{-\omega \tau_{j}} \int_{d / \tau_{j}}^{D / h_{j}} e^{-c \tau_{j} x} x^{-1} d x \leq C L e^{-\omega \tau_{j}},
$$

for $\kappa \nu>0$ sufficiently small, with the same $\omega$ as above.

Finally, it follows from (101) and HR2 that for $\kappa \nu>0$ sufficiently small,

$$
\left|R\left(h_{l} z\right)\right| \leq e^{-\omega h_{l}}, \quad l=1, \ldots, j-1 \quad \text { for } z \in \Gamma_{3}^{(j)} .
$$

Combined with the evident inequality

$$
\left|R\left(h_{j} z\right)-R_{\infty}\right| \leq C e^{-\nu h_{j}}\left(1+h_{j}|z+\nu|\right)^{-1} \quad \text { for } z \in \Gamma_{3}^{(j)},
$$

this yields by HA2,

$$
\left\|I_{3}\right\| \leq C L e^{-\omega \tau_{j}} \int_{D / h_{j}}^{\infty}\left(1+h_{j} x\right)^{-1} x^{-1} d x \leq C L e^{-\omega \tau_{j}} .
$$

Now, since $\left|R_{\infty}\right| \leq e^{-\kappa \nu}$ for $\kappa \nu>0$ sufficiently small, altogether (97), (100), (102), and (103) imply (95). This completes the proof. 
Remark 4.1. If in the statement of Theorem 4.1, $\kappa \nu>0$ is not sufficiently small, one can achieve this by reducing $\nu$ in HA1. Therefore, the above argument shows that in this case, the estimate (93) is still valid with some $\omega \in(0, \nu)$ not necessarily close to the original $\nu$.

Theorem 4.2. Let the operator $A$ and the function $R(z)$ fulfil the hypotheses HA2 with $\nu>0, L>0, \chi \in(0, \pi / 2)$ and HR1, HR2, HR4 with $\varphi \geq \chi, p \geq 1$. Let $\mathcal{T}$ be a set of stepsize sequences satisfying hypothesis $\mathbf{H S} 1$ and let $\alpha>0$ be such that (77) holds. Then for any $K_{n} \subset K \in \mathcal{T}$,

$$
\left\|R\left(K_{n} ; A\right)\right\| \leq C L\left(1+t_{n}^{\alpha}\right)^{-1} .
$$

We remark again that for methods with $R_{\infty}=0$, the number $\alpha>0$ in (104) can be chosen as large as desired.

Proof. As above, it suffices to show (104) with $H_{n}$ in place of $K_{n}$, where $H_{n}$ is the ordered sequence for $K_{n}$.

Let $\gamma_{0}$ be a fixed positive number. Given $n$, let $j_{n}$ be the index defined by (83) and (84). Further let the numbers $\tau_{j}, d, D$ and the contour $\Gamma^{(j)}$ be just the same as in the proof of Theorem 4.1. We choose $\gamma_{0}$ sufficiently large to achieve that

$$
\delta_{0}=\sup _{\operatorname{Re} z \leq-\gamma_{0}+d}|R(z)|<Q^{-\alpha},
$$

with $Q$ given by HS1. Our proof of (104) is based on the identity

$$
\begin{aligned}
R\left(H_{n} ; A\right)= & \sum_{j=j_{n}+1}^{n} R_{\infty}^{j-j_{n}-1}\left(R\left(h_{j} A\right)-R_{\infty} I\right) \\
& \times \prod_{l=j+1}^{n} R\left(h_{l} A\right) \prod_{q=1}^{j_{n}} R\left(h_{q} A\right)+R_{\infty}^{n-j_{n}} \prod_{q=1}^{j_{n}} R\left(h_{q} A\right) .
\end{aligned}
$$

Let the contours $\widetilde{\Gamma}^{(j)}, \widetilde{\Gamma}_{q}^{(j)}, q=1,2,3$ coincide with the contours $\Gamma^{(j)}, \Gamma_{q}^{(j)}, q=$ $1,2,3$, respectively, with $\tau_{j_{n}}+h_{j}$ substituted for $\tau_{j}$. We have the representation for $j=j_{n}+1, \ldots, n$,

$$
\begin{aligned}
& \left(R\left(h_{j} A\right)-R_{\infty} I\right) \prod_{l=j+1}^{n} R\left(h_{l} A\right) \prod_{q=1}^{j_{n}} R\left(h_{q} A\right) \\
& \quad=\frac{1}{2 \pi i} \int_{\tilde{\Gamma}^{(j)}}\left(R\left(h_{j} z\right)-R_{\infty}\right) \prod_{l=j+1}^{n} R\left(h_{l} z\right) \prod_{q=1}^{j_{n}} R\left(h_{q} z\right)(z I-A)^{-1} d z .
\end{aligned}
$$

In a manner similar to that used in the proof of Theorem 4.1 we get with some $\omega \in(0, \nu]$ sufficiently small,

$$
\left|R\left(h_{l} z\right)\right| \leq\left\{\begin{array}{cll}
e^{-\omega h_{l}} e^{C|z+\nu| h_{l}} & \text { if } & z \in \widetilde{\Gamma}_{1}^{(j)}, \\
e^{-\omega h_{l}} e^{-c|z+\nu| h_{l}} & \text { if } & z \in \widetilde{\Gamma}_{2}^{(j)}, \\
e^{-\omega h_{l}} & \text { if } & z \in \widetilde{\Gamma}_{3}^{(j)},
\end{array}\right.
$$

for $l=1, \ldots, j_{n}$, and

$$
\left|R\left(h_{j} z\right)-R_{\infty}\right| \leq\left\{\begin{array}{cll}
C & \text { if } & z \in \widetilde{\Gamma}_{1}^{(j)}, \\
C e^{-c|z+\nu| h_{j}} & \text { if } & z \in \widetilde{\Gamma}_{2}^{(j)}, \\
C\left(1+h_{j}|z+\nu|\right)^{-1} & \text { if } & z \in \widetilde{\Gamma}_{3}^{(j)},
\end{array}\right.
$$


for $j=j_{n}+1, \ldots, n$. Moreover, we have

$$
\left|R\left(h_{j} z\right)\right| \leq \delta_{0}, \quad z \in \widetilde{\Gamma}_{(j)}, \quad j=j_{n}+1, \ldots, n .
$$

By (107)-1109) and the argument applied in the proof of Theorem 4.1 it follows from (106) that for $j=j_{n}+1, \ldots, n$,

$$
\left\|\left(R\left(h_{j} A\right)-R_{\infty} I\right) \prod_{l=j+1}^{n} R\left(h_{l} A\right) \prod_{q=1}^{j_{n}} R\left(h_{q} A\right)\right\| \leq C L \delta_{0}^{n-j+1} e^{-\omega \tau_{j_{n}}} .
$$

By Remark 4.1 we obtain as well

$$
\left\|\prod_{q=1}^{j_{n}} R\left(h_{q} A\right)\right\| \leq C L e^{-\omega \tau_{j_{n}}}
$$

where without loss of generality we can assume $\omega$ to be just the same as in (110). In view of the inequality $\left|R_{\infty}\right| \leq \delta_{0}$, combining (105), (110), and (111) thus yields

$$
\left\|R\left(H_{n} ; A\right)\right\| \leq C L\left(n-j_{n}\right) \delta_{0}^{n-j_{n}} e^{-\omega \tau_{j_{n}}},
$$

and since $\delta_{0} Q^{\alpha}<1$,

$$
\left\|R\left(H_{n} ; A\right)\right\| \leq C L Q^{-\left(n-j_{n}\right) \alpha} e^{-\omega \tau_{j_{n}}}
$$

It remains, as in the proof of Theorem 3.2 , to combine this with (76) and (75).

Theorem 4.3. Let the operator $A$ and the function $R(z)$ satisfy the conditions of Theorem 4.2. If $K=\left(k_{j}\right)_{j=1}^{\infty}$ is a stepsize sequence for which $\lim _{n \rightarrow \infty} t_{n}=\infty$, then

$$
\lim _{n \rightarrow \infty}\left\|R\left(K_{n} ; A\right)\right\|=0 \text {. }
$$

Proof. Note that the estimate (112) is obtained without any restrictions on the stepsize sequence. Therefore, the result follows by using the same argument as in the proof of Theorem 3.3

Acknowledgments. Figure 1] was prepared with the help of the Geneva Group Graphics package GGGraphics. We are grateful to Ernst Hairer for introducing us to this package.

\section{REFERENCES}

[1] N. Bakaev, Stability estimates for a certain general discretization method, Dokl. Akad. Nauk SSSR 309 (1989), 11-15 (in Russian); English transl. in Soviet Math. Dokl. 40 (1990). MR 91e:65104

[2] N. Bakaev, Some problems of well-posedness of difference schemes on nonuniform grids, Zhurn. Vychisl. Mat. i Mat. Fiz. 33 (1993), 561-577 (in Russian); English transl. in Comput. Math. Math. Phys. 33 (1993). MR 94g:65085

[3] N. Bakaev, On variable stepsize Runge-Kutta approximations of a Cauchy problem for the evolution equation, BIT 38 (1998), 462-485. MR 99i:65069

[4] Ph. Brenner and V. Thomée, On rational approximations of semigroups, SIAM J. Numer. Anal. 16 (1979), 683-694. MR 80j:47052

[5] Ph. Brenner, V. Thomée, and L. Wahlbin, Besov Spaces and Applications to Difference Methods for Initial Value Problems, Lecture Notes in Mathematics 434, Springer-Verlag, Berlin, 1975. MR 57:1106

[6] E. Hairer and M. Zennaro, On error growth functions of Runge-Kutta methods, Appl. Numer. Math. 22 (1996), 205-216. MR 97j:65116

[7] G. H. Hardy, J. E. Littlewood, G. Pólya, Inequalities, Cambridge University Press, Cambridge, 1988. MR 89d:26016 
[8] E. Hille and R. Phillips, Functional Analysis and Semigroups, AMS, Providence, 1957. MR 19:664d

[9] M.-N. LeRoux, Semidiscretizations in time for parabolic problems, Math. Comp. 33 (1979), 919-931. MR 80f:65101

[10] Ch. Lubich and O. Nevanlinna, On resolvent conditions and stability estimates, BIT 31 (1991), 293-313. MR 92h:65145

[11] Ch. Lubich and A. Ostermann, Hopf bifurcation of reaction-diffusion and Navier-Stokes equations under discretization, Numer. Math. 81 (1998), 53-84. MR 2000m:37176

[12] A. Lunardi, Analytic Semigroups and Optimal Regularity in Parabolic Problems, Birkhäuser, Basel, 1995. MR 96e:47039

[13] C. Palencia, On the stability of variable stepsize rational approximations of holomorphic semigroups, Math. Comp. 62 (1994), 93-103. MR 94c:47066

Department of Mathematics, Air Force Technical University, Planetnaya 3, Moscow 125190, RUSSIA

E-mail address: bakaev@math.unige.ch, bakaev@postman.ru

Section de mathématiques, Université de Genève, C.P. 240, CH-1211 Genève 24, SWITZERLAND

E-mail address: Alexander.Ostermann@math.unige.ch 